\title{
Canonical Correlation of 3D Visual Fatigue between Subjective and Physiological Measures
}

\author{
Myeung Ju Won ${ }^{1}$, Sang In Park ${ }^{1}$, Mincheol Whang ${ }^{2}$ \\ ${ }^{1}$ Department of Emotion Engineering, The University of Sangmyung, Seoul, 110-743 \\ ${ }^{2}$ Department of Digital Media, The University of Sangmyung, Seoul, 110-743
}

\begin{abstract}
Objective: The aim of this study was to investigate the correlation between 3D visual fatigue and physiological measures by canonical correlation analysis enabling to categorical correlation. Background: Few studies have been conducted to investigate the physiological mechanism underlying the visual fatigue caused by processing 3D information which may make the cognitive mechanism overloaded. However, even the previous studies lack validation in terms of the correlation between physiological variables and the visual fatigue. Method: 9 Female and 6 male subjects with a mean age of $22.53 \pm$ 2.55 voluntarily participated in this experiment. All participants were asked to report how they felt about their health sate at after viewing 3D. In addition, Low \& Hybrid measurement test(Event Related Potential, Steady-state Visual Evoked Potential) and for evaluating cognitive fatigue before and after viewing 3D were performed. The physiological signal were measured with subjective fatigue evaluation before and after in watching the 3D content. For this study suggesting categorical correlation, all measures were categorized into three sets such as included Visual Fatigue set(response time, subjective evaluation), Autonomic Nervous System set(PPG frequency, PPG amplitude, HF/LF ratio), Central Nervous System set(ERP amplitude $\mathrm{P} 4, \mathrm{O} 1, \mathrm{O} 2$, ERP latency P4, O1, O2, SSVEP S/N ratio P4, O1, O2). Then the correlation of three variables sets, canonical correlation analysis was conducted. Results: The results showed a significant correlation between visual fatigue and physiological measures. However, different variables of visual fatigue were highly correlated to respective HF/LF ratio and to ERP latency(O2). Conclusion: Response time was highly correlated to ERP latency $(\mathrm{O} 2)$ while the subjective evaluation was to HF/LF ratio. Application: This study may provide the most significant variables for the quantitative evaluation of visual fatigue using HF/LF ratio and ERP latency based human performance and subjective fatigue.
\end{abstract}

Keywords: Canonical correlation analysis, 3D visual fatigue, Physiological signals

\section{Introduction}

2009년 영화 '아바타(Avatar)'의 흥행 성공 이후, 사람들 의 $3 \mathrm{D}$ 에 대한 관심은 급속도로 높아졌다. $3 \mathrm{D}$ 산업이 다시 각광받게 되면서 $3 \mathrm{D}$ 영상 처리와 $3 \mathrm{D}$ 디스플레이 기술의 빠 른 발전으로 현재는 차세대 방송 기술로 자리매김하고 있다.
최근 이러한 기술의 진보와 함께 $3 \mathrm{D}$ 시청이 유발하는 시각 적 피로감과 같이 시청자의 안전성과 관련된 영상 안전성 이슈가 제기되고 있다. 이는 $3 \mathrm{D}$ 산업의 활성화를 위해서 반드시 해결되어야 할 문제다(Hoffman et al., 2008). 국제 표준화 기구 ISO는 2005년 영상물의 시각적 피로와 관련 하여 안전성에 주목한 국제 협의안(IWA3; International Workshop Agreement)을 제안하였다. 이는 광 과민성 발작

Corresponding Author: Mincheol Whang. Sangmyung University, 7 Hongji-Dong, Jongno-Gu, Seoul 110-743.

Mobile: +82-10-2386-5293, E-mail: whang@smu.ac.kr

Copyright@2012 by Ergonomics Society of Korea(pISSN:1229-1684 eISSN:2093-8462). All right reserved.

(c) This is an open-access article distributed under the terms of the Creative Commons Attribution Non-Commercial License(http://creativecommons.org/licenses/by-nc/3.0/), which permits unrestricted non-commercial use, distribution, and reproduction in any medium, provided the original work is properly cited. http://www.esk.or.kr 
(photosensitive epileptic seizures), 시각유도멀미(visually induced motion sickness), 시각피로(visual fatigue)를 포 함한 콘텐츠 제공에서 고려해야 할 시청자의 안전성 측면 확보를 부각하고 있다(IWA3:ISO, 2005). 이처럼 3D 디스 플레이를 통하여 콘텐츠를 시청할 때 발생하는 피로감은 안 구통증을 수반하는 시각적 피로감과 이중상 경험, 두통, 멀 미, 어지러움 증상을 동반하고 심한 경우 구토를 유발하기도 한다. 이와 같은 현상들을 흔히 3D 피로라 한다(IWA3:ISO, 2005). 이와 같이 시청자에게 발생하는 3D 인체 안전성 문 제의 원인은 어디로부터 기인하는지에 대해 과학적인 연구 또는 조사를 해보기 전에는 명확히 답변하는 것에 어려움이 있다. 현 상황에서 분명한 것은 $3 \mathrm{D}$ 콘텐츠 시청 시 시각피 로의 문제가 존재한다는 점에 있다 $(\mathrm{Li}, 2010 \mathrm{a} ; \mathrm{Li} 2010 \mathrm{~b})$.

$3 \mathrm{D}$ 시각피로(Visual Fatigue)는 3D 콘텐츠를 시청할 때 가장 빈번하게 발생하며 결코 무시할 수 없는 비율로 보고되 고 있다. 이에 따라 시청자의 안전 시청을 확보하기 위하여 휴먼팩터 분야의 표준규격을 만들 때 반드시 포함되어야 할 것들로 피로도 및 품질 측정도구와 안전 시청 가이드라인, 콘텐츠 제작 가이드 라인을 요구하고 있으며 이 중 가장 기 본적인 것은 피로도 및 품질 측정 방법의 표준화 이다. 즉, $3 \mathrm{D}$ 시각피로나 품질에 영향을 미치는 파라미터 값들을 적절 한 수준으로 유지할 것을 요구하는 것이 표준안에 포함되어 야 할 내용이므로 $3 \mathrm{D}$ 시각피로나 품질을 측정하는 방법이 먼저 개발되고 최우선적으로 고려해야 하는 것이 논리적으 로 타당하다. 따라서 $3 \mathrm{D}$ 시각피로가 어떠한 특정 조건에서 증가하고 감소하는지에 대한 근본적인 원인을 확인하고 이 를 최소화 하기 위해 시각피로를 유발시킬 수 있는 세부유 발요인에 대한 연구가 진행되어야 한다. 그러나 세부유발요 인을 규명하기 위해서는 $3 \mathrm{D}$ 시각피로를 정량적으로 평가할 수 있는 방법론적 연구가 선행되어야 한다. 따라서 $\mathrm{ERP}$ (Event Related Potential) 등의 중추신경계의 인지 반응 이나, PPG(Photoplethysmogram), GSR (Galvanic skin response), SKT(Skin temperature) 등의 자율신경계 반 응 그리고 눈 깜박임(Blinking rate) 등의 시각 기능을 통해 시각피로를 정량적으로 평가하기 위한 연구가 진행되고 있 다(Table 1).

Sumio Yano 등(2002)의 연구에서는 주관설문을 통해 $3 \mathrm{D}$ 영상 시청에 의한 시각피로의 정도를 평가 했다. Park 등 $(2011 \mathrm{a}, 2011 \mathrm{~b})$ 의 연구에서는 3D 콘텐츠 시청으로 유발 되는 시각피로가 자율신경계 기능에 미치는 영향을 확인하 고, 이러한 자율신경계 영향이 교감 및 부교감신경계와 심혈 관계 반응에 어떠한 영향을 나타나는지 확인하였다. Trejo 등 (2007)의 연구에서는 뇌의 인지 반응 관점에서 접근하여 자발 뇌파를 통해 시각피로를 확인하였다. Li 등(2008)의 연구에서는 유발 뇌파를 통하여 시각피로 발생 시 $\mathrm{ERP}$ 의
Table 1. Visual fatigue measurement and evaluation division

\begin{tabular}{c|c}
\hline \multirow{4}{*}{$\begin{array}{c}\text { Subject } \\
\text { evaluation }\end{array}$} & $\begin{array}{c}\text { Author \& Year / Point } \\
\text { Li, 2010; Kuze et al., 2008; Kooi et al., 2004; } \\
\text { Hakkinen et al., 2002; Iwakiri et al., 2004 }\end{array}$ \\
\cline { 2 - 2 } & $\begin{array}{c}\text { In general, Visual fatigue evaluated using } \\
\text { subjective test about Before and After viewing } \\
\text { content for about few hour }\end{array}$ \\
\hline \multirow{2}{*}{$\begin{array}{c}\text { Autonomic } \\
\text { nervous } \\
\text { system }\end{array}$} & $\begin{array}{c}\text { Heo et al., 2010; Park et al., 2011; } \\
\text { Naschitz et al., 2002; }\end{array}$ \\
\cline { 2 - 2 } Kaneko, K. and Sakamoto, K., 2001 \\
\hline \multirow{2}{*}{$\begin{array}{c}\text { Central } \\
\text { nervous } \\
\text { system }\end{array}$} & $\begin{array}{c}\text { Li et al., 2008; Trejo, 2007; Ishigure et al., 2004; } \\
\text { Lee et al., 2010; Lambooij et al., 2009; } \\
\text { Nichols, 1999; Heo et al., 2008 }\end{array}$ \\
\cline { 2 - 2 } & Component: ERP(P700), SSVEP \\
\hline \multirow{2}{*}{ Vision } & $\begin{array}{c}\text { Lee, 2011; Miyao et al., 1989; } \\
\text { Nakaishi et al., 1999; Kaneko et al., 2001 }\end{array}$ \\
\cline { 2 - 2 } & Blinking rate, Pupil size \\
\hline
\end{tabular}

P700이 나타나는 반응을 보고하였다. Mun 등(2012)의 연 구에서는 고차원적 인지 반응을 나타내는 $\mathrm{ERP}$ 와 저차원적 인지 반응의 지표라 할 수 있는 SSVEP(Steady-state visual evoked potential)를 $3 \mathrm{D}$ 콘텐츠 시청 전과 후에 동 시에 측정하여 인지피로를 평가하였다. 또한 Lee(2011)의 연구에서는 눈 깜박임과 동공 조절 속도를 통한 눈 영상을 촬영하여 시각피로를 평가하였다.

이처럼 $3 \mathrm{D}$ 콘텐츠 시청으로 인해 유발되는 시각피로는 단 순 시각적 눈의 피로감에서부터 뇌의 정보처리과정의 과부 하 발생으로 유발된 인지적 피로까지 여러 관점에서 연구가 진행되고 있다. 하지만 이렇게 다양한 센서기반의 생체 변수 를 통하여 시각피로를 측정하려는 노력에 비해 생체 변수와 시각피로간 어떠한 영향력이 미치는지에 대하여 실재 확인 한 연구는 아직 미비하다. 이에 따라 생체 신호의 자율신경 계와 중추신경계에 따라서 주관적 시각피로와 인지작업 수 행도에 미치는 영향력이 다를 수 있으며, 이들 변수 집단간 변수 유형에 따라 차이가 있을 수 있다. 즉, 그룹간 유의성을 분석해야만 주관적 시각피로 및 인지작업 수행도와 생리 신 호간의 관계성을 알 수 있다. 이를 위해 다수의 그룹간 상관 성 분석이 가능한 정준상관분석 방법을 이용한 상관성 분석 이 필요하다. 따라서 본 연구에서는 $3 \mathrm{D}$ 콘텐츠 시청으로 유 발되는 시각피로에 대한 생체 변수 집단간 상관성을 확인하 기 위해 정준상관분석을 시행하여 시각피로에 의한 주관적 느낌과 인지작업 수행도의 영향을 비교하였다. 또한 구체적 으로 피로 변수들 사이의 상호관계 구조 분석을 실시하여 
시각피로 평가에 사용되는 생체 신호들이 주관적 시각피로 와 인지작업 수행도에 따라 차이가 있는지 확인하고, 생체 신호의 자율신경계 변수와 중추신경계 변수 집단간 변수들 사이에서 시각피로와 최대 상관성을 나타내는 생체 신호를 확인하여 좀더 정교하고 상관성 높은 시각피로의 생리적 반 응을 규명하고자 한다.

\section{Method}

\subsection{Participants}

본 연구에 참여한 피실험자는 시력(또는 교정시력이) 0.8 이상인 15명(여 9명; 평균 나이 22.53세 \pm 2.55 )의 입체 시 정상인을 대상으로 하였다. 실험에 참가한 모든 피험자는 실 험 전날 카페인, 음주, 알코올 섭취를 금하도록 하고 충분한 수면을 유지하여 실험 전 피로를 최소화 할 수 있도록 요청 받았다. 그리고 모든 피험자로부터 연구목적을 제외하고 실 험에 대한 대략적인 사항에 대해 설명한 후 자발적 의지에 대한 피험자 동의서를 얻었다. 또한 참여도를 높이기 위하여 실험에 참여한 대가로 소정의 금액을 지불하였다.

\subsection{Experimental design}

기존 선행 연구를 기반으로 시각피로와 관련하여 생체 신호를 수집하기 위한 실험을 진행하였으며 실험 절차는 Figure 1과 같다.

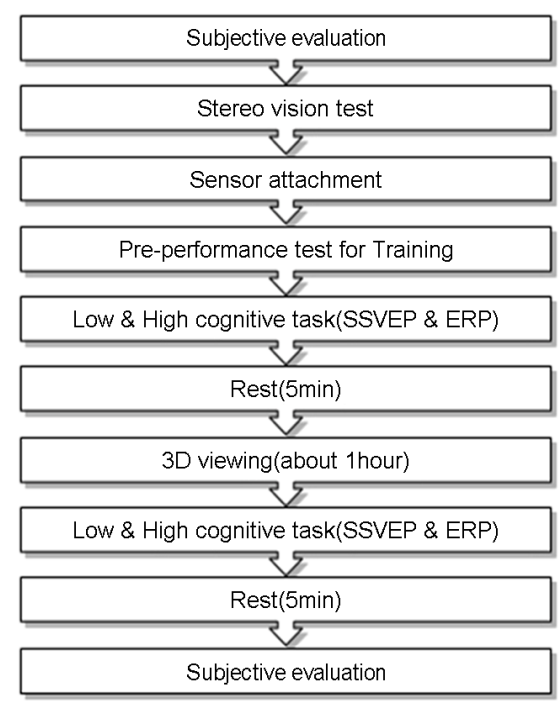

Figure 1. Experimental procedure used in this study
피험자는 $\mathrm{Li}$ 등(2010c)이 개발한 주관적 피로 평가 측정 도구를 사용하여 피험자의 상태에 대한 설문을 시청 전과 시청 후에 5점 척도로 보고 받았다. 또한 Cho 등(2011)이 개발한 입체 시 테스트 프로그램을 이용하여 피험자가 3D 입체 영상에 대한 깊이감을 지각하는지에 대하여 평가하였 다. 피험자는 편안한 상태에서 $3 \mathrm{D}$ 영상을 1 시간 동안 시청 하였으며, 시청 전과 시청 후에 생체 신호를 측정하였다. 신 호는 자율신경계의 대표 반응 지표인 맥파(PPG, PhotoPlethysmoGram), 피부전도반응(GSR, Galvanic Skin Response) 및 피부온도(SKT, Skin temperature) 와 인지 반응을 나타내는 중추신경계의 사건관련전위(ERP, EvokedRelated Potential) 와 시유발지속전위 (SSVEP, Steady State Visually Evoked Potential)를 측정하여 수집하였다. 주관 평가 설문지는 시각적 스트레스, 눈 통증, 신체통증, 상 흐림 의 4 가지 요인으로 구성되어 있으며, 역 문항을 포함하여 주 관 평가 데이터의 신뢰성을 확보하였다. 인지 반응 측정은 선행 연구를 기반으로 SSVEP와 $\mathrm{ERP}$ 를 동시에 측정하기 위하여 표준자극 11 개(A부터 $\mathrm{K}$ 까지) 와 표적자극 1 개(숫자 $5)$ 의 총 12 개로 구성되어 랜덤하게 제시되었다. 이때 피험 자의 시선을 화면 중앙에 고정한 채로 집중을 지시된 화살 표 방향으로 하게 하였으며, 인지작업 수행도에 따른 타겟에 대한 반응시간을 기록하였다(Mun et al., 2012). 실험에 사 용된 3D 입체 영상은 입체감이 뛰어난 것으로 알려진 라푼 젤(The Walt Disney Company, 2010)을 사용하여 40inch LED 3D TV(Un40D6400, Samsung)를 통해 제시하였다.

\subsection{Data acquisition and extract effective parameters}

본 연구에서는 선행 연구를 기반으로 시각피로와 관련하 여 유효한 생체 변수들을 추출하였다. 이에 자율신경계의 대표 반응인 맥파(PPG), 피부전도반응(GSR), 피부온도 (SKT)를 통해 시각피로를 측정한 연구(Park et al., 2011a) 와 PPG 신호에서 PRV (Pulse rate Variability) 분석을 통 해 교감(LF, Low frequency), 부교감(HF, High frequency) 반응을 확인한 연구(Park et al., 2011b) 및 인지피로 관 점에서 사건관련전위 $(\mathrm{ERP})$ 를 통해 시각피로를 측정한 연 구가 있다(Mun et al., 2012). 상기의 연구를 통해 PPG frequency, PPG amplitude, HF/LF ratio, ERP amplitude (P4, O1, O2), ERP latency (P4, O1, O2), SSVEP S/N $\operatorname{ratio}(\mathrm{P} 4, \mathrm{O} 1, \mathrm{O} 2)$ 의 생체 신호 변수가 시각피로 측정에 유 효한 파라미터임을 확인하였다. 또한 이에 따른 시각피로 발 생 여부를 확인하기 위한 지표로 주관적 피로 평가 설문지를 통한 주관 평가와 인지작업 수행도에 따른 타겟에 대한 반 응시간을 사용하였으며, 이를 피로와 비피로 그룹을 구분 하는데 사용하였다(Mun et al., 2012; Park et al., 2011a; 
Park et al., 2011b).

Table 2. Prior research result used in this study

\begin{tabular}{|c|c|c|c|c|}
\hline $\begin{array}{l}\text { Advanced } \\
\text { research }\end{array}$ & \multicolumn{2}{|c|}{$\begin{array}{c}\text { Effective } \\
\text { parameters }\end{array}$} & Result & $p$-value \\
\hline $\begin{array}{c}\text { Cardiovascular } \\
\text { system } \\
\text { (Park et al., } \\
\text { 2011a) }\end{array}$ & \multicolumn{2}{|c|}{$\mathrm{HF} / \mathrm{LF}$ ratio } & Decrease & .005 \\
\hline \multirow{4}{*}{$\begin{array}{l}\text { Autonomic } \\
\text { nervous } \\
\text { system } \\
\text { (Park et al., } \\
\text { 2011b) }\end{array}$} & \multicolumn{2}{|c|}{ PPG frequency } & Increase & .005 \\
\hline & \multicolumn{2}{|c|}{ PPG amplitude } & Decrease & .005 \\
\hline & \multicolumn{2}{|c|}{ GSR mean } & Increase & .005 \\
\hline & \multicolumn{2}{|c|}{ SKT mean } & Decrease & .005 \\
\hline \multirow{9}{*}{$\begin{array}{c}\text { Central } \\
\text { nervous } \\
\text { system } \\
\text { (Mun et al., } \\
\text { 2012) }\end{array}$} & \multirow{3}{*}{$\begin{array}{c}\text { ERP } \\
\text { amplitude }\end{array}$} & $\mathrm{P} 4$ & Decrease & .013 \\
\hline & & $\mathrm{O} 1$ & Decrease & .006 \\
\hline & & $\mathrm{O} 2$ & Decrease & .004 \\
\hline & \multirow{3}{*}{$\begin{array}{l}\text { ERP } \\
\text { latency }\end{array}$} & $\mathrm{P} 4$ & Increase & .037 \\
\hline & & $\mathrm{O} 1$ & Increase & .027 \\
\hline & & $\mathrm{O} 2$ & Increase & .002 \\
\hline & \multirow{3}{*}{$\begin{array}{l}\text { SSVEP } \\
\text { S/N ratio }\end{array}$} & $\mathrm{P} 4$ & Decrease & .010 \\
\hline & & O1 & Decrease & .050 \\
\hline & & $\mathrm{O} 2$ & Decrease & .010 \\
\hline
\end{tabular}

\section{Analysis}

\subsection{Canonical correlation analysis}

$3 \mathrm{D}$ 콘텐츠 시청으로 유발된 시각피로에 의한 주관적 느낌 과 인지작업 수행도 영향과 유효 생체 변수 집단간의 구조적 분석을 목적으로 Hotelling(1935)에 의해 제안된 정준상관 분석(Canonical correlation analysis)을 수행하였다(Lee, 2002). 정준상관분석은 두 개 이상의 기준 변수(multiple criterion variable)와 두 개 이상의 설명 변수(multiple predictor variable) 들 사이의 상호관계를 연구하는 다변량 분석기법이다.

이에 따라 본 연구에서는 상기 연구를 통해 확인한 주관 평가 결과와 타겟에 대한 반응시간 결과를 반영하는 인지 작업 수행도를 시각피로 변수 집단으로 설정하였으며, $\mathrm{PPG}$ frequency, PPG amplitude, HF/LF ratio는 자율신경계 변수 집단으로 설정하였고, $\mathrm{ERP}$ amplitude $(\mathrm{P} 4, \mathrm{O} 1, \mathrm{O} 2)$, ERP latency (P4, O1, O2), SSVEP S/N ratio (P4, O1, O2) 를 중추신경계 변수 집단으로 설정하였다. 정준상관분석의 경우는 두 변수간 어떠한 영향을 미치는가를 확인하고자 하
는 경우가 대부분이기 때문에 이때의 기준 변수와 설명 변수 는 분석의 편의를 위해 용어 상으로만 구분된다.

15 명의 피험자(여 9 명; 평균 나이 22.53세 \pm 2.55 ) 데이 터를 기반으로 SPSS $17.0 \mathrm{~K}$ 를 사용하여 분석을 실시하였다. 각각의 두 변수 집단의 선형 조합(linear combination)간의 상관관계를 가장 크게 만드는 최대 선형 조합(maximum linear combination) 을 찾아내어 시각피로 변수 집단과 자율 신경계 및 중추신경계의 변수 집단 사이의 상관성을 확인하 였고, 다수의 변수들간의 상관관계를 통하여 각 변수 집단에 포함된 개별 변수의 정보를 확인 후 두 변수 집단간의 상관 계수가 최대가 되게 하는 변수를 확인하였다. 또한 산출된 결과는 표준정준가중치 (standardized canonical weights) 와 정준교차적재량(canonical cross-loading)에 기초하여 해석 하였다.

\section{Results}

\subsection{Visual fatigue and autonomic nervous system}

시각피로군과 자율신경계 변수 집단 사이의 정준상관분 석 결과는 Table 3 와 Table 4 과 같다. 도출된 2 개의 정준 상관함수 중 첫 번째 함수에서 유의한 결과가 나타났다 $(p$ $=.048)$. 표준정준가중치에서 함수 1 의 주관적 시각피로가

Table 3. Result of canonical correlation analysis with ANS(PPG, PRV) \& VF

\begin{tabular}{c|c|c|c|c}
\hline \multirow{2}{*}{} & \multicolumn{2}{|c|}{ Function1 } & \multicolumn{2}{c}{ Funcion2 } \\
\cline { 2 - 5 } & $\mathrm{W}$ & $\mathrm{C}-\mathrm{L}$ & $\mathrm{W}$ & $\mathrm{C}-\mathrm{L}$ \\
\hline 1 Set(Visual fatigue) & \multicolumn{4}{|c}{-} \\
\hline Response time & .223 & .452 & 1.077 & .398 \\
\hline Subjective evaluation & .887 & .749 & -.650 & -.100 \\
\hline 2 Set(ANS) & \multicolumn{5}{|c}{-} \\
\hline PPG frequency & .110 & .161 & 1.046 & .195 \\
\hline PPG amplitude & -.414 & -.515 & -.435 & -.126 \\
\hline HF/LF ratio & -.660 & -.575 & -.303 & .013 \\
\hline
\end{tabular}

W: canonical weight, C-L: canonical cross-loading

Table 4. Result of canonical correlations and redundancy measure of shared variance(VF \& ANS)

\begin{tabular}{l|c|c|c|c}
\hline & C-C & $R_{r d} 1$ & $R_{r d} 2$ & sig \\
\hline Function 1 & .765 & .654 & .363 & .048 \\
\hline Function 2 & .494 & .346 & .097 & .228 \\
\hline
\end{tabular}

C-C: canonical correlations, $R_{r d}$ : redundancy index 
0.887로 높게 나타났으며, $\mathrm{HF} / \mathrm{LF}$ ratio가 -0.660 임을 확인 하였다. 또한 정준교차적재량은 주관적 시각피로에서 0.749 , PPG amplitude에서 -0.515 , HF/LF ratio에서 -0.575 를 나타내었다.

\subsection{Visual fatigue and central nervous system(ERP amplitude)}

시각피로군과 중추신경계(ERP amplitude)의 변수 집단 사이의 정준상관분석 결과는 Table 5 와 같다. 도출된 2 개의 정준상관함수 모두 $(p>.05)$ 통계적으로 유의하지 않은 결과 가 도출되었다.

Table 5. Result of canonical correlations and redundancy measure of shared variance(VF \& CNS: ERP amplitude)

\begin{tabular}{l|c|c|c|c}
\hline & $\mathrm{C}-\mathrm{C}$ & $R_{r d} 1$ & $R_{r d} 2$ & $\operatorname{sig}$ \\
\hline Function 1 & .646 & .494 & .641 & .221 \\
\hline Function 2 & .217 & .506 & .313 & .714 \\
\hline
\end{tabular}

C-C: canonical correlations, $R_{r d}$ : redundancy index

\subsection{Visual fatigue and central nervous system(ERP latency)}

시각피로군과 중추신경계(ERP latency)의 변수 집단 사 이의 정준상관분석 결과는 Table 6와 Table 7 과 같다. 도 출된 2 개의 정준상관함수 중 첫 번째 함수에서 유의한 결과 가 나타났다 $(p=.013)$. 표준정준가중치에서 함수 1 의 반 응시간이 -1.032 로 높게 나타났으며, ERP latency (O2)가 -3.281 임을 확인하였다. 또한 정준교차적재량은 반응시 간에서 -0.997 , ERP latency $\mathrm{O} 1$ 지점에서 $-0.659, \mathrm{ERP}$

Table 6. Result of canonical correlation analysis with CNS(ERP latency) \& VF

\begin{tabular}{c|r|r|r|r}
\hline \multirow{2}{*}{} & \multicolumn{2}{|c|}{ Function1 } & \multicolumn{2}{c}{ Funcion2 } \\
\cline { 2 - 5 } & $\mathrm{W}$ & \multicolumn{1}{c}{$\mathrm{C}-\mathrm{L}$} & $\mathrm{W}$ & $\mathrm{C}-\mathrm{L}$ \\
\hline 1 Set(Visual datigue) & \multicolumn{4}{|c}{-} \\
\hline Response time & -1.032 & -.997 & -.379 & .077 \\
\hline Subjective evaluation & .084 & -.345 & 1.096 & .939 \\
\hline 2 Set(CNS) & \multicolumn{5}{|c}{-} \\
\hline P4 latency & 1.401 & -.670 & .143 & -.502 \\
\hline O1 latency & 1.157 & -.659 & -3.084 & -.699 \\
\hline O2 latency & -3.281 & -.823 & 2.351 & -.461 \\
\hline W: cancy
\end{tabular}

W: canonical weight, C-L: canonical cross-loading
Table 7. Result of canonical correlations and redundancy measure of shared variance(VF \& CNS: ERP latency)

\begin{tabular}{c|c|c|c|c}
\hline & $\mathrm{C}-\mathrm{C}$ & $R_{r d} 1$ & $R_{r d} 2$ & sig \\
\hline Function 1 & .807 & .557 & .520 & .013 \\
\hline Function 2 & .309 & .443 & .318 & .496 \\
\hline
\end{tabular}

C-C: canonical correlations, $R_{r d}$ : redundancy index

latency P4 지점에서 -0.670, ERP latency $\mathrm{O} 2$ 지점에서 -0.823 순으로 높게 나타났다.

\subsection{Visual fatigue and central nervous system(SSVEP $\mathrm{S} / \mathrm{N}$ ratio)}

시각피로군과 중추신경계(SSVEP S/N ratio)의 변수 집단 사이의 정준상관분석은 다음과 같다(Table 8). 도출된 2 개 의 정준상관함수에서 정중상관계수 모두 $(p>.05)$ 통계적인 유의차를 확인할 수 없었다.

Table 8. Result of canonical correlations and redundancy measure of shared variance(VF \& CNS: SSVEP S/N ratio)

\begin{tabular}{c|c|c|c|c}
\hline & Wilk's & Chi-SQ & DF & sig \\
\hline Function 1 & .490 & 9.994 & 6.000 & .125 \\
\hline Function 2 & .825 & 2.696 & 2.000 & .260 \\
\hline
\end{tabular}

$\mathrm{W}$ : canonical weight, C-L: canonical cross-loading

\section{Conclusion}

본 연구에서는 $3 \mathrm{D}$ 콘텐츠 시청으로 유발된 시각피로에 대 한 생체 변수를 확인하고 정준상관분석을 이용하여 시각피 로 평가에 사용되는 생체 신호들이 실재 시각피로에 의한 주관적 느낌과 인지작업 수행도에 따라서 차이가 있는지 확 인하고자 하였다. 또한 둘 사이의 상호 관련이 있을 경우 최 대의 상관성을 나타내는 생체 신호를 확인하고자 하였다. 이 를 통해, 시각피로와 유효 생체 신호로 확인된 자율신경계 변수 집단과 중추신경계 변수 집단 사이의 밀접한 관계가 있 다는 결론을 도출하였다. 또한 정준교차적재량 결과 주관적 시각피로도가 증가할수록 $\mathrm{HF} / \mathrm{LF}$ ratio와 $\mathrm{PPG}$ amplitude가 감소하는 경향을 나타내었으며, $3 \mathrm{D}$ 인지피로를 반영하는 반 응시간이 증가할수록 $\mathrm{ERP}$ latency의 $\mathrm{O} 2$ 지점에서 감소하 는 경향을 나타내었고 ERP latency의 $\mathrm{P} 4$ 지점과 $\mathrm{O} 1$ 지점 또한 영향력이 큰 것으로 판단되었다. 이는 주관적 시각피로 
의 경우 자율신경계 변수와 큰 상관성이 있으며, 중추신경계 의 경우 인지적 피로를 반영하는 인지작업 수행도와 큰 상 관성이 있음을 의미한다. 또한, 두 변수 집단의 상관관계가 최대가 되게 하는 변수 집단간의 선형조합 계수를 정준가중 치라 하며 이러한 각 변수들을 표준화시킨 다음 구해진 표 준정준가중치를 통해서 자율신경계 변수 중 $\mathrm{HF} / \mathrm{LF}$ ratio와 중추신경계 변수 중 ERP latency $(\mathrm{O} 2)$ 가 시각피로에 가장 큰 영향을 미치는 것을 확인하였다. 이는 자율신경계 변수인 $\mathrm{HF} / \mathrm{LF}$ ratio가 PPG frequency와 PPG amplitude 보다 시 각피로와 높은 상관성이 있음을 의미하고, 중추신경계 변수 인 ERP latency의 $\mathrm{P} 4$ 지점과 $\mathrm{O} 1$ 지점보다 $\mathrm{O} 2$ 지점에서 시각피로와 높은 상관성이 있다고 해석할 수 있다. 본 연구 결과에 따라, $\mathrm{HF} / \mathrm{LF}$ ratio와 ERP latency $(\mathrm{O} 2)$ 가 시각피로 의 생리적 반응을 규명하기 위한 좀더 정교하고 상관성 높 은 유효 생체 변수 임을 확인하였다.

본 연구에서는 주관적 시각피로도는 자율신경계의 $\mathrm{HF} / \mathrm{LF}$ ratio에서 가장 큰 상관성이 나타남을 확인하였다. 이는 사 람들이 느끼는 주관적인 시각피로감에 따라 자율신경계의 부교감신경 활성도가 감소하고 교감신경 활성도가 증가되어 교감신경계가 항진되는 결과에 영향이 있는 것으로 해석할 수 있다. 또한 타겟에 대한 반응시간을 반영하는 인지작업 수행도에 따라 ERP latency $(\mathrm{O} 2)$ 에서 높은 상관성을 나타 내는 결과를 확인하였다. 이는 중추신경계의 후두엽 부분의 $\mathrm{O} 2$ 지점에서 $\mathrm{ERP}$ latency가 증가하는 것으로 $\mathrm{BD}$ 시청으로 인하여 뇌의 정보처리과정의 과부하의 발생으로 유발된 인 지적 피로에 의해서 타겟에 대한 반응시간에 영향력이 기여 한다는 의미로 해석할 수 있다.

한편 본 연구는 상관 연구이기 때문에 상기 연구에서 확인 한 변수들간의 관계를 인과적으로 해석하기에는 한계가 있 다. 따라서 추후 연구에서는 본 연구의 한계점을 보완하여 생체 신호 변수 집단간의 관계를 확인해볼 필요성이 있다. 본 연구는 $3 \mathrm{D}$ 콘텐츠에 따른 시각피로에 의한 주관적 느낌 과 인지작업 수행도에 따라서 생체 변수간 실재 상관성이 있 는지 확인하였으며, 생체 신호의 자율신경계 변수 집단과 중 추신경계 변수 집단간 변수들 사이에서 시각피로와 최대 상 관성을 나타내는 생체 신호를 확인하고, 좀더 정교한 상관성 이 높은 시각피로의 생리적 반응을 확인해 본 연구로서 그 의의가 있을 것으로 생각된다.

\section{Acknowledgements}

This work was partly supported by the IT R\&D program of MKE/KEIT [2012-10038745，3D Equipment/Device Centered
"Human Factor" Research and Development for Securing Human Safety].

\section{References}

Cho, S. J., Han, H. N., Lee, H. D. and Park, M. C., "Development of Stereopsis Test Program for Measurement of Awareness Ratio of Stereoscopic Image", Conference on The Korean society of broadcast engineers, 2011.

Hakkinen, J., Vuori, T. and Paakka, M., "Postural stability and sickness symptoms after HMD use", The Proceedings of IEEE International Conference on Systems, Man and Cybernetics, 147-152, 2002.

Heo, H., Lee, E. C., Woo, J. C., Kim, C. J., Park, K. R. and Whang, M. C., A realistic game system using Multi-modal user interface, IEEE Transactions on Consumer Electronics, 53(6), 1364-1372, 2010.

Hoffman, D. M., Girshick, A. R., Akeley, K. and Banks, M. S., Vergenceaccommodation conflicts hinder visual performance and cause visual fatigue, Journal of Vision, 8(33), 1-30, 2008.

Ishigure, Y., Suyama, S., Takaha, H., Nakazawa, K., Hosohata, J., Takao, Y. and Fujikado, T., Evaluation of visual fatigue relative in the viewing of a depth-fused 3-D display and 2-D display, Proc Int Diso Worksjops, 11, 1627-1630, 2004.

Iwakiri, K., Mori, I., Sotoyama, M., Horiguchi, K., Ochiai, T., Jonai, H. and Saito, S., Survey on visual and musculoskeletal symptoms in VDT workers, Journal of Occupational Health, 46(6), 201-212, 2004.

IWAS : ISO, International Workshop Agreement 3, 2005.

Kaneko, K. and Sakamoto, K., Spontaneous blinks as criterion of visual fatigue during prolonged work on visual display terminals, Perceptual and Motor Skills, 92(1), 234-250, 2001.

Kooi, F. L. and Toet, A., Visual comfort of binocular and 3D displays, Displays, 25(2-3), 99-108, 2004.

Kuze, J. and Ukai, K., Subjective evaluation of visual fatigue caused by motion images, Displays, 29(2), 2008.

Lambooij, M. and Ijsselsteijn, W., Visual Discomfort and Visual Fatigue of Steroscopic Display: A Review, Journal of Imaging Science and Technology, 53(3), 030201-1-14, 2009.

Lee, E. C., Camera Vision Based System for Quantitatively Measuring Emotional Responses Caused by Visual Stimuli, In Proceeding of Korean Workshop on Image Processing and Image Understanding, to be appeared. 2011.

Lee, Y. J., Understanding of the Canonical Correlation Analysis, 2002.

Li, H.-C. O., 3D Human Factors: Steps to standardizing Guidelines for Human-friendly 3D, The Korean Information Display Society, 27(3), 36-41, 2010a.

Li, H.-C. O., Human Factor Research on the Measurement of Subjective Three Dimensional Fatigue, The Korean Information Display Society, 15(5), 607-616, 2010b

Li, H.-C. O., Human Factor Research on the Measurement of Subjective Three Dimensional Fatigue, The Korean society of broadcast engineers, 
15(5), 607-706, 2010c.

Li, H.-C. O., Seo, J. H., Kham, K. T. and Lee, S. H., Measurement of 3D Visual Fatigue Using Event-Related Potential (ERP): 3D Oddball Paradigm, 3DTV Conference: The True Vision - Capture, Transmission and Display of 3D Video, 213-216, 2008.

Lichols, M. and Newsome, W., The Neurobiology of cognition, Nature (London), 402(6761), 35-38, 1999.

Miyao, M., Hacisalihzade, S. S. and Allen J. S., Effects of VDT resolution on eyestrain and readability: an eye movement approach, Ergonomics, 32(6), 603-614, 1989.

Mun, S. C., Park, M. C., Park S. I. and Whang, M. C., SSVEP and ERP measurement of cognitive fatigue caused by stereoscopic 3D, Neuroscience Letters in press, 525(2), 89-94, 2012.

Nachitz, J. E., Sabo, E., Naschitz, S., Rosner, I., Rozenbaum, M., Priselac, R. M., Gaitini, L., Zukerman, E. and Teshurun, D., Fractal analysis and recurrence quantification analysis of hert rate and pulse transit time for diagnosing chronic fatigue syndrome, Clinical Autonomic Research, 12(4), 1619-1560, 2002.

Nakaishi, H. and Yamada, Y., Abnormal tear dynamics and symptoms of eyestrain in operators of visual display terminals, Occupational and Environmental Medicine, 56, 6-9, 1999.

Park, S. I., Whang, M. C., Kim, J. H., Mun, S. C. and Ahn, S. M., Autonomic Nervous System response affected by 3D visual fatigue evoked during watching 3D TV, The Korean Society for Emotion \& Sensibility, 14(4), 653-662, 2011a.

Park, S. I., Whang, M. C., Kim, J. H., Mun, S. C. and Ahn, S. M., Autonomic Nervous System response affected by 3D visual fatigue evoked during watching 3D TV, The Korean Society for Emotion \& Sensibility Conference, $2011 \mathrm{~b}$.

Takahashi, M., "LCD vs PDP picture quality status and the task of FPD TVs", The Korean Display Conference COEX, 2006.

Trejo, L. J., Knuth, K., Prado, R., Rosipal, R., Kubitz, K., Kochavi, R., Mattews, B. and Shang, T., EEG-Based Estimation of Mental Fatigue: Convergent Evidence for a Three-State Model, Lecture Notes in Computer Science, 201-211, 2007.

Yano, S., Ide, S., Mitsuhashi, T. and Thwaites, H., A study of visual fatigue and visual comfort for 3D HDTV/HDTV images, Displays, 23(4), 191-201, 2002.
Yano, S., Wmoro, M. and Mitsuhashi, T., Two factors in visual fatigue caused by stereoscopic HDTV images, Displays, 25(4), 141-150, 2004.

\section{Author listings}

Myeung Ju Won: dnjsaudwn@naver.com

Highest degree: B.S., Division of Multi Media, National Institute for Lifelong Education(NILE)

Position title: M.S. student, Department of Emotion Engineering, Sangmyung University

Areas of interest: 3D Human Factors, Emotion engineering, $\mathrm{HCl}$

Sang In Park: ini0630@naver.com

Highest degree: B.S., Division of Oriental Health Care Herbal Pharmaceutical Science, Joongbu University

Position title: M.S. student, Department of Emotion Engineering, Sangmyung University

Areas of interest:3D Human Factors, Emotion engineering, BCI

Mincheol Whang: whang@smu.ac.kr

Highest degree: Ph.D., Biomedical Engineering, Georgia Institute of Technology

Position title: Professor, Division of Digital Media, Sangmyung University

Areas of interest: Human factors, Emotion engineering, BCI and Neurocardiology

Date Received : 2012-10-22

Date Revised : 2012-11-30

Date Accepted : 2012-12-02 